\title{
Modeling and Simulation of Hydrothermal Oxidation of Organic Compounds
}

\author{
S. Vielcazals, J. Mercadier, and F. Marias \\ Laboratoire de Thermique Energétique et Procédés (LaTEP), Ecole Nationale Supérieure d'Ingénieurs en Génie des \\ Technologies Industrielles, Université de Pau et des Pays de l'Adour -EA 1932, Rue Jules Ferry, B.P. 7511,64075 Pau \\ Cedex, France \\ D. Matéos, M. Bottreau, and F. Cansell \\ Institut de Chimie de la Matière Condensée de Bordeaux (ICMCB), Université Bordeaux 1, 87, avenue Dr Schweitzer, 33608 \\ Pessac Cedex, France

\section{Marraud} \\ SNPE Matériaux Energétiques, Avenue Gay-Lussac, BP 57, 33166 Saint Médard en Jalles Cedex, France
}

\begin{abstract}
Hydrothermal oxidation is an efficient and clean way for the treatment of wastewater containing organic matter. The purpose of this work is to develop a mathematical model of a reactor for hydrothermal oxidation. This reactor is horizontal and tubular and is designed with multi-injection points of oxidant. Its diameter is very small with regard to its length. The mathematical model is based on the plug flow assumption. The governing equations are: momentum, mass, species, and energy balances. Thanks to this model, the profiles of temperature, and concentration of chemical species are computed along the reactor. The numerical predictions of the model are compared to experimental profiles obtained in the case of supercritical oxidation of methanol. These comparisons show very good agreement.
\end{abstract}

Keywords: hydrothermal oxidation, modeling, reactor, experimental device

\section{Introduction}

In the context of sustainable development, it is required that industrial wastes undergo elimination and/or valorization treatments. The cost of these treatments increases unceasingly because of the environmental protection norms. An important part of these industrial wastes is mainly composed of water and is indexed as aqueous wastes. When their organic content is more than one percent, they are too concentrated to undergo a biological treatment, while when it is less than 10 percent, their Correspondence concerning this article should be addressed to
frederic.marias@univ-pau.fr. treatment by incineration is too expensive because it requires extra gas.

Hydrothermal oxidation in supercritical water is an alternative way to dispose of these effluents. The main advantage of that technology is that the by-products of the process are nontoxic. Indeed, carbon is oxidized into carbon dioxide and hydrogen into water, while hetero-atoms are converted into mineral salts. ${ }^{1-4}$ Moreover, this technology allows for the recovery of an important part of the energy released by the exothermic reaction of oxidation.

The basic principle of this process is to oxidize organic matter into carbon dioxide and water, and inorganic material into mineral acids or oxides, using a supercritical fluid as the reacting medium. In the frame of this study, this medium is supercritical water, this means water above its critical point 


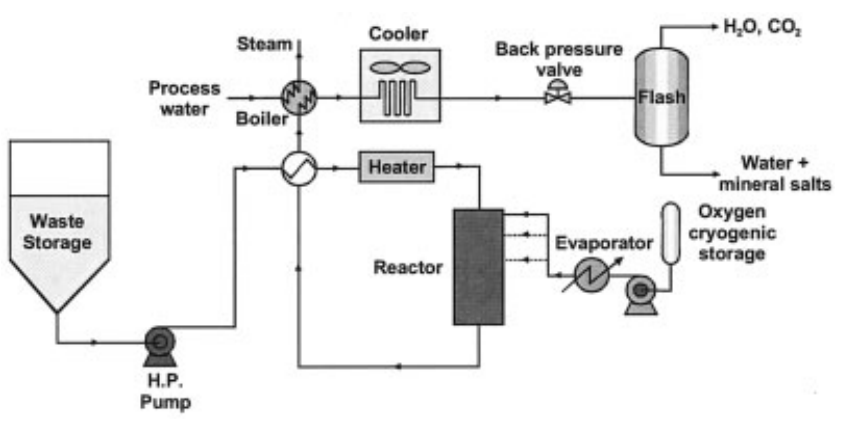

Figure 1. Supercritical water oxidation process.

(22.06MPa, 647.13K). Figure 1 sketches such a process. Aqueous waste is pumped from storage, raised to $25 \mathrm{MPa}$, and preheated to approximately $673 \mathrm{~K}$. It is then supplied to a chemical reactor where it is mixed with oxygen in order to be converted to carbon dioxide and water. The mixture leaving the reactor is cooled before entering a back pressure valve where pressure is dropped. The expended fluid then enters a flash where gaseous and liquid components are separated.

The Institut de Chimie de la Matière Condensée de Bordeaux (ICMCB) research program in this field focuses on two major parts. The first one is devoted to the study of chemical pathways and kinetic parameters. The second one is devoted to the development of new reactors. In order to improve the waste treatment by hydrothermal oxidation from a technical and economic point of view, the ICMCB and the Hydrothermal Oxidation Option (HOO) Company have developed a horizontal and tubular reactor. Its main feature is that oxidant is injected at three different locations along the reactor in order to decrease temperature levels inside the reactor (which in turn enhances the control of the oxidation reactions) and to avoid thermal runaways. ${ }^{5,6}$

The design of such reactors is a complex task because of the numerous physical and chemical phenomena that occur within the devices under consideration. To succeed in the design procedure, an experimental apparatus, at laboratory scale, has been elaborated by the ICMCB. The first part of this article focuses on the description of this device. Experiments performed on this reactor yield a lot of information on the transformation of the aqueous waste. However, because of the high-pressure conditions that prevail, it is very difficult to access physical data inside the reactor. A mathematical model can be an efficient way to tackle this problem. Indeed, once validated by comparisons with experiments, such a model can provide further data and especially data concerning the inner part of the reactor. Moreover, once validated, such a model might be very useful for the scale up of supercritical reactors at industrial scale. That is why the Laboratoire de Thermique Energétique et Procédés (LaTEP) has developed such a model. The second part of this article is devoted to the description of the assumptions and the governing equations that were used to elaborate this mathematical model.

The last part of this article shows comparisons of profiles of temperature along the reactor obtained on the experimental device for the one side and by the mathematical model on the other side. Those experiments were carried out using methanol as organic waste. Agreement between the two profiles is ex- cellent, especially when kinetics used in the model issued from other experiments preformed on the ICMCB device.

\section{Experimental Apparatus and Procedures}

Figure 2 sketches the experimental apparatus of the ICMCB. It is a tubular reactor made in inconel 625 . It is $42 \mathrm{~m}$ long, its internal diameter is $1.6 \mathrm{~mm}$, and its external diameter is 3.2 $\mathrm{mm}$. Three injection ports of oxygen are distributed along the reactor. The first injection is located at the inlet of the reactor, the second one at $\mathbf{1 1 . 6}$ meters downstream, and the last one at 24.1 meters from the input. The number of injectors is fixed by the chemical oxygen demand of the waste. Indeed, high values might lead to very important temperature rise near the injector if enough oxygen is brought to the reactor. These high gradients of temperature must be reduced in order to protect the material of the reactor itself. Generally, three injections are sufficient to allow for the oxidation to happen without damaging the reactor. The oxidant (pure oxygen) is injected at $25^{\circ} \mathrm{C}$. The nominal capacity of the reactor is $3 \mathrm{~kg} / \mathrm{h}$ of aqueous waste. The operating pressure is $25 \mathrm{MPa}$, and the temperature at the inlet ranges from $250^{\circ} \mathrm{C}$ to $600^{\circ} \mathrm{C}$, depending upon the power sent to the pre-heater.

This reactor is surrounded with a thermal shield. It is composed of a stainless steel shell surrounded by brand electrical heaters wrapped around the shell and calcite insulation. It is insulated on the first 34 meters. The role of the electrical heaters is twofold. When the reactor is used to determine kinetics data, it is required that the reactor is isothermal. In this case, the electrical heaters are fixed by a PID controller and their output power is variable. When the reactor is used to estimate its efficiency in terms of organic material conversion, the electrical heaters compensate the thermal losses of the apparatus, which is not strictly adiabatic. The values of the output powers are fixed by making a preliminary experiment with pure water, keeping the whole reactor at the inlet temperature of the waste under study. The three maximal output powers of the heaters are 500, 1000, and 3000 watts, respectively.

A high-pressure pump feeds the aqueous waste at $25 \mathrm{MPa}$ to an electrical pre-heater that, in turns, feeds the reactor at the desired temperature. The oxidant (here, pure oxygen) is pressurized by a Hackel compressor that injects oxygen at $25 \mathrm{MPa}$ without any preheating. In order to control the working of the reactor, and to get experimental data, 28 thermocouples are

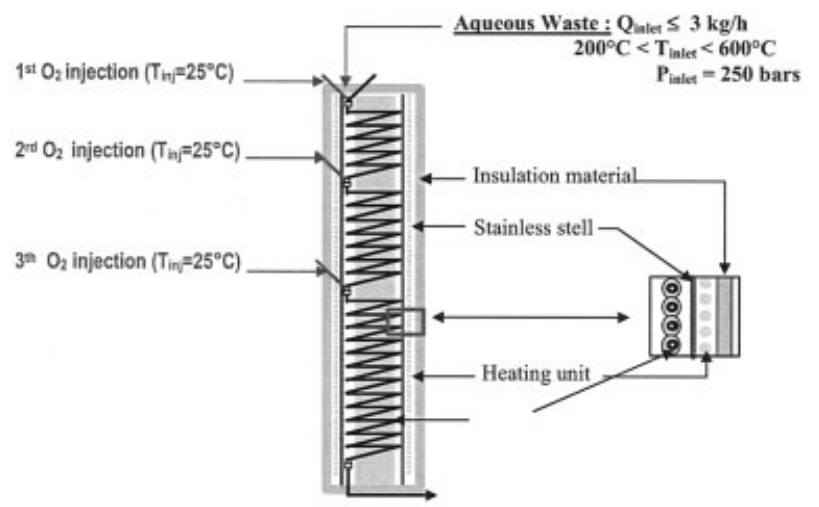

Figure 2. Sketch of the ICMCB facility. 
distributed over the surface of the reactor and 3 thermocouples can be placed inside the reactor.

The control of the whole system is performed by a computer running LabView Software.

At the reactor outlet and after temperature and pressure reduction, the gas phase composition is analyzed by gas chromatography with a thermal conductivity detector (TCD). For liquid samples, the organic amount is determined by measurement of chemical oxygen demand (COD) according to the French normalization method for water and wastewater analysis. $^{7}$

\section{Mathematical Modeling}

The building of the mathematical model describing the physical and chemical phenomena that occur within the reactor is a quite complex task. This model relies on several assumptions that need to be discussed first. Then, the governing equations of the model are presented. Finally, some insights are given on the solution procedure.

\section{Assumptions}

Inside the reactor, several species coexist. The main part of the total mass flow rate of the fluid flowing inside the reactor is water. Because of the nature of the waste itself, this water is loaded with organic compounds. Moreover, after the oxidant injection, some oxygen might be present in the mixture and also some products of reaction $\left(\mathrm{CO}_{2}\right.$, for example). Thus, the prediction of the thermodynamic properties of the mixture (enthalpy and density) should take into account the influence of these species on each other, as well as the influence of temperature and pressure. However, because of the low organic content of the waste entering the reactor (less than $10 \%$ on a mass basis), we assume that the reacting medium is pure water. (As will be seen in the Results section, the mass fraction of water is always greater than 95\%.) These properties are computed from the IAPWS formulation for pure water. ${ }^{8}$ So the first assumption is:

A1: Thermodynamical properties (specific enthalpy, density) of the reacting medium are assumed to be equal to pure water properties.

The second assumption (which is close to the first one) is relative to the transport properties of the reacting medium. Indeed, with the same justification as assumption A1, we postulate:

A2: Transport properties (thermal conductivity and viscosity) of the reacting medium are equal to pure water properties and are computed from the IAPWS formulation for pure water.

Given some general operating conditions (temperature at inlet is above $250^{\circ} \mathrm{C}$ and pressure is $25 \mathrm{MPa}$ ) and a mass flow rate of $3 \mathrm{~kg} / \mathrm{h}$, the Reynolds's number at the entrance of the reactor can be evaluated: $\mathrm{Re}=5500$. Thus, the flow-field inside the reactor is fully turbulent. That is why we assume the plug flow mode of operation of this reactor. Thus, in the stationary mode of operation, the state variables of the system are one dimensional. This leads to the third assumption:

A3: In the stationary mode of operation, the system under study is considered as one dimensional.

Dealing with industrial wastes, a lot of organic components might be present inside the effluent to clean. Moreover, these components can have very complex structures. Thus, it is very difficult to predict their reaction pathway inside the reactor. That is why we have assumed that for any species present in the waste, the reaction mechanism is lumped into the following global one step reaction:

$$
C_{\alpha} H_{\beta} O_{\gamma}+\left(\alpha+\frac{\beta}{4}-\frac{\gamma}{2}\right) \cdot \mathrm{O}_{2} \rightarrow \frac{\beta}{2} \cdot \mathrm{H}_{2} \mathrm{O}+\alpha \cdot \mathrm{CO}_{2}
$$

A4: For each organic compound taken into account, the reaction pathway is lumped into a global one step reaction.

From the kinetics point of view, we expect the rate of reaction to be dependent upon temperature, as postulated by Arrhénius. Moreover, we assumed that this rate depends on the local concentration of waste and oxygen. Thus, for any chemical compound taken into account, the rate of reaction is expressed as follows:

$$
r=k^{\circ} \exp \left(\frac{-E_{a}}{R \cdot T}\right) \cdot C_{w}^{m} \cdot C_{\mathrm{O}_{2}}^{n}
$$

In Eq. 2, $k^{0}$ stands for the pre-exponential factor of Arrhénius's law and $E_{a}$ for the activation energy of reaction (1), while $C_{w}$ and $C_{\mathrm{O}_{2}}$ represent, respectively, the local concentration of the waste under consideration and the oxygen. $m$ and $n$ stand for the order with respect to waste and oxygen.

As has been explained in the paragraph devoted to the presentation of the experimental device, three electrical heaters provide heat to the reactor. Because this power is brought to the reactor through its external surface, and because of the thickness of the reactor $(0.8 \mathrm{~mm})$, we have supposed that the conduction phenomenon inside the reactor material was acting as a "power averaging" device. Thus, if we denote by $P_{1}, P_{2}$, and $P_{3}$ the power yields, respectively, by the three electrical heaters, we postulate that the inner fluid receives a volumetric power expressed as the volume averaged power yield to the reactor.

A6: The volumetric power received by the reacting medium from the electrical heater is:

$$
w_{\text {elec }}=\frac{P_{1}+P_{2}+P_{3}}{S L}
$$

where $S$ stands for the cross sectional area of the reactor and $L$ its length.

Although the whole apparatus is thermally insulated, it is obvious that thermal losses exist. They have to be taken into account in the energy balance of the reactor. To compute these losses, we have assumed that at each location of the reactor, a specific heat flux was lost according to Newton's law for heat transfer. For convenience, we relate this external specific heat flux to a volumetric power lost by the reacting medium.

A7: The volumetric power lost by the reactor by thermal losses is expressed as:

$$
w_{\text {lost }}=\frac{H}{d}\left(T-T_{\text {ext }}\right)
$$


In this expression, $H$ represents the overall heat transfer coefficient with the surroundings, $d$ the inner diameter of the reactor, and $T$ and $T_{\text {ext }}$ stand, respectively, for the local temperature and the external temperature.

Because of turbulence phenomena, some importance mechanisms exist that might increase the rate of transport of species and energy under gradients of concentration and temperature, respectively. However, because of the high value of the ratio $L / d$, we assume the axial diffusion of species to be negligible with respect to the convective process $\left(\right.$ Villermaux $\left.{ }^{9}\right)$. Assuming further that Lewis's number equals unity, we draw the same conclusion for thermal transport processes:

A8: Axial diffusion of species and energy are negligible.

As has been pointed out in the Introduction, one of the main advantages of using supercritical water is that dissolution of oxygen is enhanced. Because, in the reactor, we are always above the critical point of pure water, and because the process operates under turbulent conditions and because of the small diameter of the injector, we assume that:

A9: Oxygen is instantaneously and completely dissolved once it is injected in the reactor.

\section{Governing equations}

One of the aims of a mathematical model is to provide more information than experimental results can yield. For example, in our case, we expect the model to be able to describe the evolution of species concentration as well as the temperature along the reactor. In order to obtain such information, we need to write some mathematical equations that translate physical and chemical phenomena into mathematical formalism. Basically, these equations postulate that, in stationary mode of operation, mass, species, momentum, and energy are conserved over a control volume of our choice. For modeling purposes, and because of the three injection ports of oxygen, the reactor has been split into three. More precisely, the inlet of each reactor is located just downstream of the injector. The boundary conditions at the inlet will be given later.

In the following paragraph we show the governing equations in their "derivative" form inside each of the three reactors.

Total Mass Conservation

$$
\frac{\partial \rho u}{\partial x}=0
$$

where $\rho$ stands for the local density of the mixture and $u$ for its velocity. This equation states that within the reactor, mass is neither created nor disappears.

\section{Momentum Conservation}

As has been quoted in assumption A3, we consider the system as one dimensional. However, a complete formulation of the momentum equation should include a two dimensional formulation of this equation because of shear stress at the wall of the reactor. Indeed, this shear stress results in high radial gradients at this location. Thus, the momentum balance is written as follows:

$$
\frac{\partial \rho u u}{\partial x}=-\frac{\partial P}{\partial x}+\frac{\partial \tau_{x j}}{\partial x_{j}}
$$

where $\mathrm{P}$ stands for the local pressure of the reacting medium, and $\tau_{x j}$ for the stress tensor, in direction $x$, linked to a gradient of velocity in the direction $j$.

Moreover, in turbulent mode of operation, the stress tensor should include the Reynolds's stress tensor and an appropriate model to compute it. This would drastically complicate the mathematical description of the process. Thus, the choice that has been made here is to compute the divergence of the stress tensor as the local pressure drop over the control volume under consideration. ${ }^{10}$ This computation has been done according to Churchill's correlation, valid for any Reynolds's number. ${ }^{11}$ The resulting balance equation is then:

$$
\frac{\partial \rho u u}{\partial x}=-\frac{\partial P}{\partial x}-\frac{\Delta \xi}{\Delta x}
$$

where $\Delta \xi / \Delta x$ represents the local lineic pressure drop inside the reactor.

\section{Species Conservation}

We use the classical formulation of chemical engineering for the description of chemical reaction. Indeed, if a set of species $J=\left\{1,2, \ldots, j, \ldots, N_{s p}\right\}$ is submitted to a set $I=\{1$, $\left.2, \ldots, i, \ldots, N_{\text {reac }}\right\}$ of chemical reactions, the total chemical reaction rate $R_{j}$ (on a mass basis) of a species $\mathrm{j}$ is linked to the rate of reactions according to:

$$
R_{j}=M_{j} \sum_{i=1}^{N_{\text {reac }}} \partial_{i, j} r_{i}
$$

where $\partial_{i, j}$ stands for the stoichiometric coefficient of species $\mathrm{j}$ in reaction $\mathrm{i}$ and $M_{j}$ for the molar weight of species $\mathrm{j}$. Given this formalism, the species balance can be written as:

$$
\frac{\partial u \cdot \rho y_{j}}{\partial x}-R_{j}=0
$$

where $y_{j}$ stands for the mass fraction of species $j$.

Energy Conservation

Following the formalism used to derive the divergence of the stress tensor, one is able to compute the dissipation (both viscous and turbulent) as ${ }^{12}$ :

$$
\Theta=u \frac{\Delta \xi}{\Delta x}
$$

Then the balance of energy over the control volume is expressed as:

$$
\frac{\partial \rho u h}{\partial x}=u \frac{\Delta \xi}{\Delta x}+u \frac{\partial P}{\partial x}+w_{\text {elec }}-w_{\text {lost }}-\sum_{i=1}^{N_{\text {reac }}} r_{i} \Delta_{r} H_{i}
$$

where $\Delta_{r} H_{i}$ stands for the standard heat of reaction $i$, and $h$ for the enthalpy to weight of the medium.

Models for Density, Enthalpy to Weight, and Viscosity

Finally, to close the problem, one needs to write equations for the computation of density and the computation of enthalpy. These equations are issued from the IAPWS formula- 


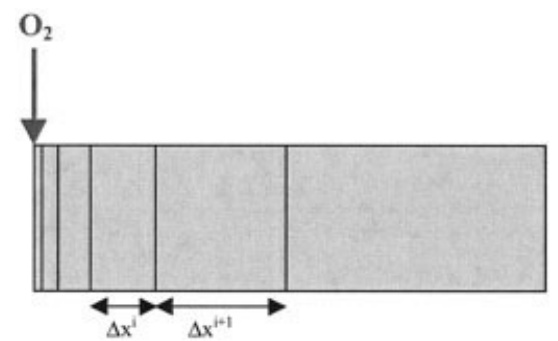

Figure 3. Illustration of the adaptive mesh used for the discretization of differential equations.

tion for pure water ${ }^{8}$ (see assumption A1) and they are written as:

$$
\begin{gathered}
\rho=\rho_{m}(P, T) \\
h=h_{m}(P, T) \\
\mu=\mu_{m}(P, T)
\end{gathered}
$$

\section{Boundary conditions}

Boundary conditions are required at the input of each of the three reactors. Because of the injection of oxygen, the conditions prevailing at the input of one reactor are not the ones that prevail at the output of the previous reactor. Indeed, because of assumption A9 (instantaneous mixing of oxygen with the supercritical fluid), the conditions at the input of the reactor are computed using a balance on an adiabatic and completely stirred tank reactor defining the injector.

If we denote $P_{\text {out }}, T_{\text {out }}, \rho_{\text {out }}, u_{\text {out }}, y_{j, \text { out }}$, and $h_{\text {out }}$ the conditions prevailing at the output of the previous reactor (or after the pre-heater of the overall process), $P_{i n}, T_{i n}, \rho_{i n}, u_{i n}, y_{j, i n}$, and $h_{i n}$ the effective conditions at the input of the reactor under consideration, and $\dot{m}_{\mathrm{O}_{2}}, u_{\mathrm{O}_{2}}$, and $h_{\mathrm{O}_{2}}$, the mass flow rate of oxygen, its velocity, and its enthalpy at the injector, then we write:

Mass balance

$$
U_{\text {in }}=\frac{\rho_{\text {out }} u_{\text {out }} S+\dot{m}_{\mathrm{O}_{2}}}{\rho_{\text {in }} S}
$$

Enthalpy balance

$$
h_{\text {in }}=\frac{\rho_{\text {out }} u_{\text {out }} h_{\text {out }} S+\dot{m}_{\mathrm{O}_{2}} h_{\mathrm{O}_{2}}}{\rho_{\text {in }} u_{\text {in }} S}
$$

Oxygen balance

$$
y_{\mathrm{O}_{2}, \text { in }}=\frac{\rho_{\text {out }} u_{\text {out }} y_{\mathrm{O}_{2}, \text { out }} S+\dot{m}_{\mathrm{O}_{2}}}{\rho_{\text {in }} u_{\text {in }} S}
$$

Species balance (except oxygen)

$$
y_{j, \text { in }}=\frac{\rho_{\text {out }} u_{\text {out }} y_{j, \text { out }}}{\rho_{\text {in }} u_{\text {in }}}
$$

\section{Momentum balance}

$$
P_{\text {in }}=\frac{\left(\rho_{\text {out }} u_{\text {out }}^{2}+P_{\text {out }}\right) S+\dot{m}_{\mathrm{O}_{2}} u_{\mathrm{O}_{2}}}{S}-\rho_{\text {in }} u_{\text {in }}^{2}
$$

In the above expressions, $\mathrm{S}$ stands for the cross sectional area of the tubular reactor.

Models for density, enthalpy to weight, and viscosity

$$
\begin{gathered}
\rho_{i n}=\rho_{m}\left(P_{i n}, T_{i n}\right) \\
h_{i n}=h_{m}\left(P_{i n}, T_{i n}\right) \\
\mu_{i n}=\mu_{m}\left(P_{i n}, T_{i n}\right)
\end{gathered}
$$

\section{Solving}

If $N_{\text {org }}$ denotes the number of organic compounds taken into account, the mathematical system under consideration is composed of $N_{\text {org }}+5$ ordinary differential equations (total mass, momentum, energy, $\mathrm{CO}_{2}, \mathrm{O}_{2}$, organics conservation) and 3 algebraic equations (model for enthalpy, density, and viscosity of the mixture). Such a system cannot be directly fed to a computer to get the proper solution. Thus, the choice has been made here to discretize the differential equations in order to get a purely algebraic system. Among the discretization methods, the finite volume method ${ }^{13}$ is very attractive because the balance equations are satisfied over each of the control volumes that constitute the mesh. That is why we have derived the algebraic equations according to the divergence formula:

$$
\int_{v} \operatorname{div}(\vec{\phi}) d V=\oint_{s} \vec{\phi} \cdot d \vec{S}
$$

where $\vec{\phi}$ stands for any vectorial data.

As will be shown in the paragraph regarding Results, the gradients of species and temperature are very important near the injections of oxygen. This stiffness of the systems requires an appropriate meshing in order to make the mathematical system more stable. To fulfill this particular topic, we have

\begin{tabular}{|c|c|c|c|c|c|c|}
\hline $\begin{array}{c}\text { Wished Temperature } \\
\left({ }^{\circ} \mathrm{C}\right)\end{array}$ & $\begin{array}{c}\text { Mass Flow Rate } \\
\text { of Water } \\
\left(\mathrm{kg} \cdot \mathrm{h}^{-1}\right)\end{array}$ & $\begin{array}{c}\text { Inlet Pressure } \\
(\mathrm{MPa})\end{array}$ & $\begin{array}{c}\text { Inlet Temperature } \\
\left({ }^{\circ} \mathrm{C}\right)\end{array}$ & $\begin{array}{l}\text { Power Sent to the } \\
\text { First Heater (W) }\end{array}$ & $\begin{array}{l}\text { Power Sent to the } \\
\text { Second Heater } \\
\text { (W) }\end{array}$ & $\begin{array}{l}\text { Power Sent to the } \\
\text { Third Heater (W) }\end{array}$ \\
\hline 350 & 1 & 24 & 352 & 75 & 75 & 150 \\
\hline 400 & 1 & 24 & 404 & 260 & 280 & 320 \\
\hline 500 & 1 & 24 & 500 & 495 & 510 & 660 \\
\hline
\end{tabular}
chosen a grid with a step size following a geometrical reason from one injector to the other:

$$
\Delta x^{i+1}=q \Delta x^{i}
$$

where $\Delta x^{i}$ stands for the size of the control volume number $i$ (Figure 3).

To conclude with the finite volume method, one needs to add an interpolation scheme allowing for prediction of the value of the state variables at the surface of the control volume as a function of the value of the state variable inside the surround-

Table 1. Operating Parameters Used for the Evaluation of $\boldsymbol{h}_{\text {ext }}$ 

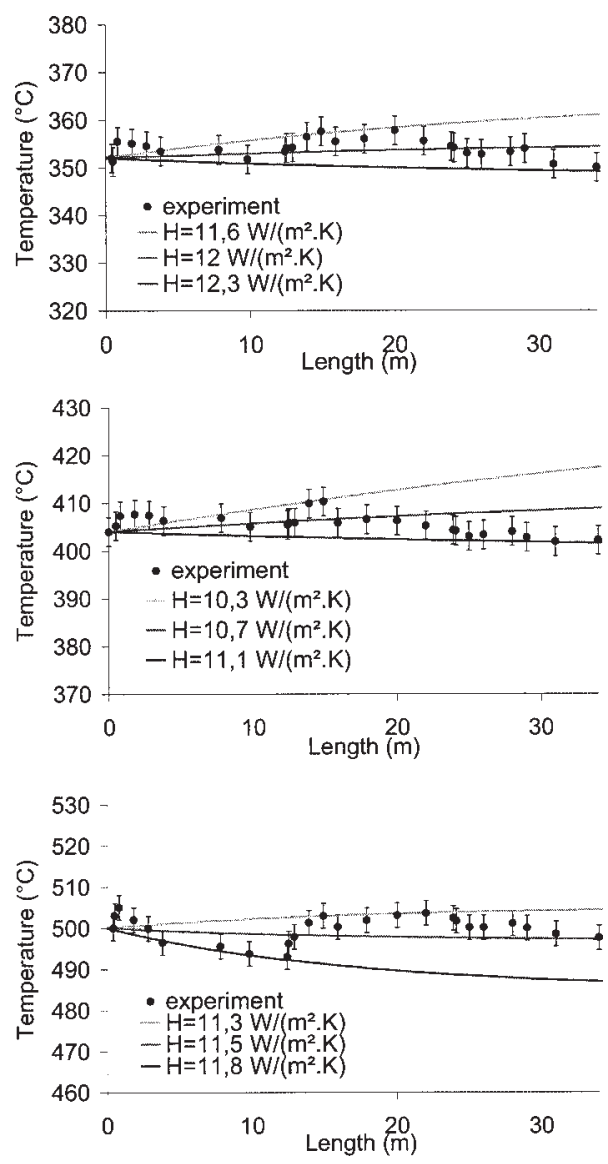

Figure 4. Comparison between experiments and numerical predictions as a function of external heat transfer coefficient. Temperature: $350^{\circ} \mathrm{C}$, $400^{\circ} \mathrm{C}$, and $500^{\circ} \mathrm{C}$.

ings control volume. Because of its better stability, we have chosen the upwind scheme for interpolation.

Finally, the algebraic system obtained after discretization is solved using Newton-Raphson's method for algebraic systems of non-linear equations.

\section{Results}

In this section, we present the results of specific experiments obtained from both the experimental and numerical points of view. Nevertheless, a value of the convective heat transfer featuring in Eq. 4 is required by the mathematical model. That is why, as preliminary results, we present an analysis that allows for the prediction of thermal losses of the device.

\section{Determination of the convective external heat transfer coefficient}

The value of this global coefficient (i.e., the thermal losses) is computed from a test with pure water, in the absence of chemical reaction and oxygen supply. This is a way to estimate heat transfer between the experimental apparatus and the surroundings. These isothermal experimental tests were carried out, at three temperatures, to show a possible modification in the properties of the insulator according to the temperature. The output powers of the electrical heaters were fitted in order to obtain the isothermal profile at the desired temperature. Then, the known values of the output powers were used in the model according to Eq. 3. The operating data are summed up in Table 1, while comparisons between experiments and prediction are shown in Figure 4. For theses numerical results, as well as the following, the first step used in the meshing scheme is $\Delta x^{0}=6.5 \times 10^{-3} \mathrm{~m}$, the geometrical reason is $q=1.01$, and 300 cells are used between two consecutive injections (total: 900 cells).

The first remark that comes about this Figure is that control of the temperature profile inside the reactor by the electrical heaters is quite good. Indeed, given the uncertainty of the thermocouples, we can assume the baseline to be isothermal.

The second remark arises from the value of the global heat transfer coefficients that have been used in order to obtain the isothermal profile from a numerical point of view. Indeed, their values are in a very narrow range (from 10.3 to 12.3 $\left.\mathrm{W} \cdot \mathrm{m}^{-2} \cdot \mathrm{K}^{-1}\right)$. Such values are very similar to the ones that can be encountered in free convection. As an average, and because the value of $H=11 \mathrm{~W} \cdot \mathrm{m}^{-2} \cdot \mathrm{K}^{-1}$ gives the best results in the case of simulation with reaction, it is kept in what follows.

\section{Oxidation of methanol}

The supercritical oxidation of methanol has been chosen for the comparison between experiment and numerical prediction. Indeed, it is possible to pre-heat this compound above the critical temperature of pure water without being damaged. Moreover, some studies have already been performed on this compound and on the same experimental design in order to determine kinetics data. ${ }^{14}$ Table 2 sums up the operating parameters that were used for this experiment (the values of the output powers of the electrical heaters were the ones used for the isothermal baseline at $450^{\circ} \mathrm{C}$ ).

Table 3 summarizes some of the data (in terms of energy of activation, $E_{a}$, as well as pre-exponential factor, $k_{0}$ ) available from literature. ${ }^{14-17}$ The authors publish their kinetics results with a range of uncertainty. For our simulations, we have chosen the value that is shown in bold character in Table 3 . Indeed, this value was used because this was the one that fitted best the experimental results and that was in the confidence

Table 2. Operating Parameters Used for the Oxidation of Methanol

\begin{tabular}{|c|c|c|c|c|c|c|c|c|c|}
\hline $\begin{array}{c}\text { Initial Mass } \\
\text { Fraction of } \\
\text { Methanol } \\
(\%)\end{array}$ & $\begin{array}{l}\text { Mass Flow } \\
\text { Rate of } \\
\text { Waste } \\
\left(\mathrm{kg} \cdot \mathrm{h}^{-1}\right)\end{array}$ & $\begin{array}{c}\text { Inlet } \\
\text { Pressure } \\
\text { (MPa) }\end{array}$ & $\begin{array}{c}\text { Inlet } \\
\text { Temperature } \\
\left({ }^{\circ} \mathrm{C}\right)\end{array}$ & $\begin{array}{c}\text { Mass Flow } \\
\text { Rate of } \mathrm{O}_{2} \\
1^{\text {st }} \text { Inj. } \\
\left(\mathrm{g} \cdot \mathrm{h}^{-1}\right)\end{array}$ & $\begin{array}{l}\text { Mass Flow } \\
\text { Rate of } \mathrm{O}_{2} \\
2^{\text {nd }} \text { Inj. } \\
\left(\mathrm{g} \cdot \mathrm{h}^{-1}\right)\end{array}$ & $\begin{array}{c}\text { Mass Flow } \\
\text { Rate of } \mathrm{O}_{2} \\
3^{\mathrm{rd}} \text { Inj. } \\
\left(\mathrm{g} \cdot \mathrm{h}^{-1}\right)\end{array}$ & $\begin{array}{l}\text { Power Sent } \\
\text { to the First } \\
\text { Heater (W) }\end{array}$ & $\begin{array}{l}\text { Power Sent } \\
\text { to the } \\
\text { Second } \\
\text { Heater (W) }\end{array}$ & $\begin{array}{l}\text { Power Sent } \\
\text { to the } \\
\text { Third } \\
\text { Heater (W) }\end{array}$ \\
\hline 1.94 & 1 & 24 & 456 & 10 & 10 & 21 & 100 & 300 & 540 \\
\hline
\end{tabular}


Table 3. Review of Kinetics Parameters for Oxidation of Methanol from Literature

\begin{tabular}{|c|c|c|c|c|}
\hline$k^{\circ}(\mathrm{mol} / \mathrm{l})^{1-m-n} / \mathrm{s}$ & $\mathrm{Ea}(\mathrm{kJ} / \mathrm{mol})$ & $\begin{array}{c}\text { Waste's } \\
\text { Order }\end{array}$ & $\begin{array}{l}\text { Oxygen's } \\
\text { Order }\end{array}$ & References \\
\hline $10^{28.8}$ & $\begin{array}{l}447.3 \pm 125.4 \\
\mathbf{4 1 7 . 3}\end{array}$ & 0.89 & 0.12 & [15] \\
\hline $10^{26.2}$ & $\begin{array}{l}408.4 \pm 85.3 \\
\mathbf{3 7 2 . 4}\end{array}$ & 1 & 0 & {$[15]$} \\
\hline $10^{24.4}$ & $\begin{array}{l}395 \pm 33 \\
\mathbf{3 6 2}\end{array}$ & 1 & 0 & [16] \\
\hline $2.51 \times 10^{29}$ & $\begin{array}{l}478.6 \\
\mathbf{4 7 8 . 6}\end{array}$ & 1 & 0 & [17] \\
\hline $6.7 \times 10^{12}$ & $\begin{array}{l}203 \pm 30 \\
\mathbf{1 8 3}\end{array}$ & 1 & 0 & {$[14]$} \\
\hline $4.7 \times 10^{7}$ & $\begin{array}{l}125 \pm 8 \\
130\end{array}$ & 1 & 0.31 & [14] \\
\hline $2.4 \times 10^{11}$ & $\begin{array}{l}175 \pm 29 \\
190\end{array}$ & 1 & 0.4 & {$[14]$} \\
\hline
\end{tabular}

range defined by the authors. Because the experimental device can provide temperature profile only, we have chosen this particular state variable to make the comparison between experiments and simulations. Figure 5 shows this comparison in the case of references ${ }^{15-17}$, while Figure 6 shows this comparison with the results of Mateos et al. ${ }^{14}$

From the experimental point of view, these Figures give insights into the process of oxidation itself. Indeed, the three rises of temperature that occur near the injections show that the oxidation is very rapid, at least in the case of methanol. This proves that the "actives regions" of the reactor are very narrow. Moreover, it can be seen that the slopes of the increase in temperature diminish from the first injection to the third, while the temperature of the reacting medium is continuously rising. This indicates that the reaction is extremely dependent upon the local concentration of organics because this concentration decreases from one injection to another. Finally, these Figures (5 and 6) also show that the multi injection procedure allows for a better control of the temperature inside the reactor. Using this mode of introduction for the oxygen, we can see that the temperature levels are confined and that it is possible to cool the reactive medium between the two injections if it is required.

From the validation point of view, we can see that the numerical predictions fit quite well the experimental results. This conclusion should, however, be moderated in the case of

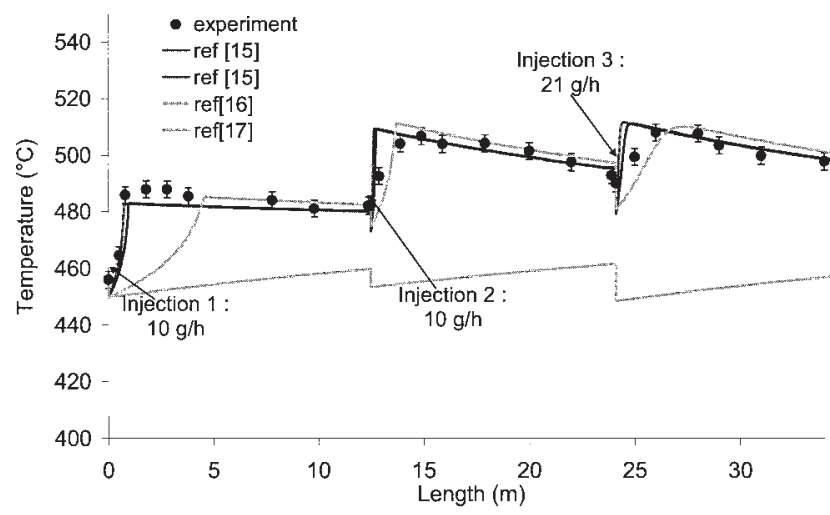

Figure 5. Comparison (in terms of temperature profile) between experiments and simulation for the oxidation of methanol. Kinetics data from ${ }^{15-17}$.

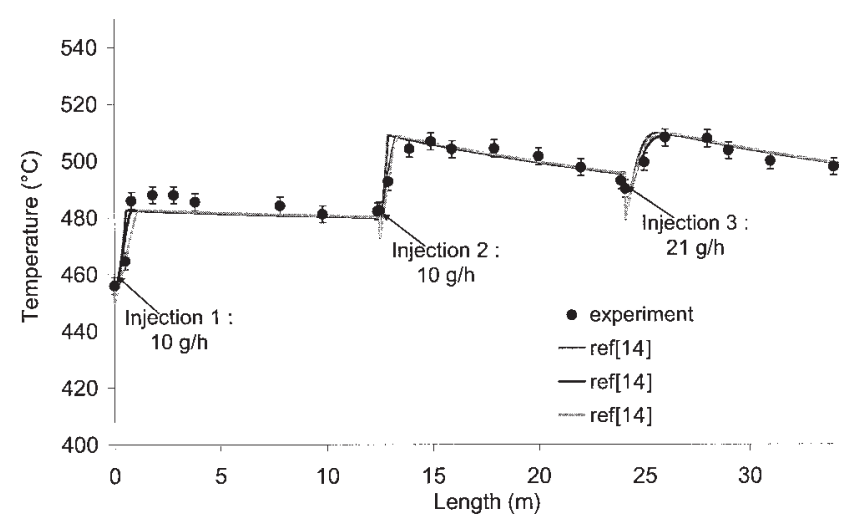

Figure 6. Comparison (in terms of temperature profile) between experiments and simulation for the oxidation of methanol. Kinetics data from ${ }^{14}$.

Webley et al., ${ }^{17}$ where the high value of the activation energy given by Webley and Tester seems to be too high for ignition of the reaction to occur. To a lower extent, the value of the kinetics parameter published by Rofer and Streit ${ }^{16}$ does not allow for a good prediction of the temperature profile near the first injection point. However, their parameters seem to give better results near the third injection. The data yield by Mateos et al. (Figure 6) allows for the best numerical prediction. This is probably linked to the experimental procedure they have followed to obtain these data. Indeed, they have used the ICMCB facilities, and have computed their kinetics parameters by making isothermal experiments at several organic and oxygen concentrations.

Given the good prediction of the temperature profile inside the reactor, we assume the model to be validated. Hence, to refine the analysis of what happens inside the reactor, Figure 7 shows the profile of mass fraction of species into the reactor. As has been quoted in the preceding paragraph, oxidation of methanol is very rapid. The depletion of oxygen downstream of the injector is very important and demonstrates that this is the limiting reactant. This also demonstrates the advantage of a multi-injection system.

\section{Conclusion}

A mathematical model for the oxidation of organics compounds into supercritical water has been presented in this

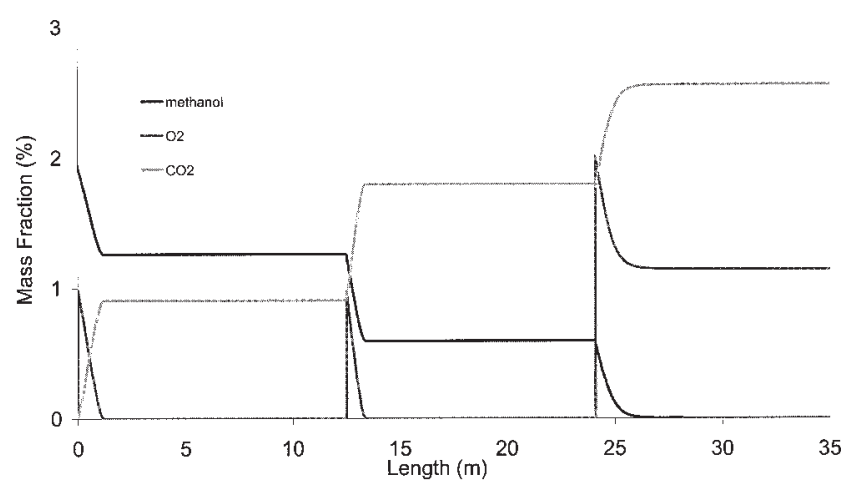

Figure 7. Profile of mass fraction of species inside the reactor. Kinetics data from ${ }^{14 c}$ 
paper. This model relies on several assumptions that have been discussed above. This model is based on the conservation of total mass, chemical species, momentum, and energy written in a steady state mode of operation. It allows for prediction of temperature and species profiles that give a detailed description of the phenomena occurring within such a reactor. The results of this model have been compared to experimental data coming from ICMCB facilities, in the case of the oxidation of methanol. As has been discussed, the numerical prediction highly depends upon the kinetics data of the reaction itself. However, this model gives excellent prediction of the temperature profile along the reactor. This indicates that the different assumptions that have been formulated are valid. Thus, this model can be used in order to give more information than the experimental apparatus can yield and also for the scaling up of supercritical water oxidation reactors.

\section{Notation}

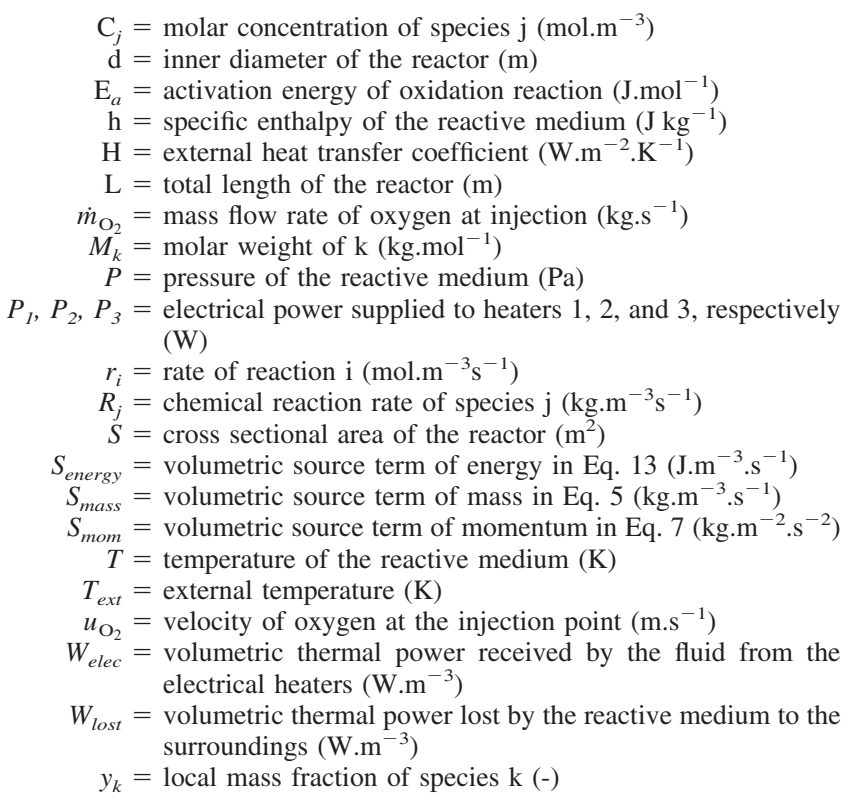

\section{Greek letters}

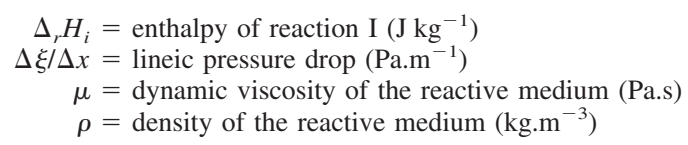

$\tau_{x j}=$ stress tensor in direction $\mathrm{x}$ linked to velocity gradient in direction $\mathrm{j}$

\section{Literature Cited}

1. Dutournié, P. Modélisation de réacteurs d'oxydation de déchets organiques en milieu aqueux supercritique. $\mathrm{PhD}$ Thesis, Université de Pau et des Pays de l'Adour, 2000.

2. Cansell F, Rey S, Beslin P. Thermodynamic aspect of supercritical fluids processing: applications to polymers and wastes treatment. $R e-$ vue de l'Institut Français du Pétrole. 1998;53:71-98.

3. Cansell F, Beslin P, Berdeu B. Hydrothermal oxidation of model molecules and industrial wastes. Environmental Progress. 1998;17: 240-245.

4. Tester JW, Holgate HR, Amellini FJ, Webley PA, Killilea WR, Hong GT, Barner HE. Supercritical water oxidation technology. Process development and fundamental research. ACS Symp Ser. 1993;518:3576.

5. Bottreau M. Hydrothermal oxidation: a new concept for treatment of industrial and urban liquid wastes. In: Bonnaudin N, Cansell F, Biarritz FO, eds. Supercritical Fluids and Materials. Institut National Polytechnique de Lorraine; Paris: 2003:369-384.

6. Cansell F. Method for Treating Waste by Hydrothermal Oxidation. CNRS Patent - WO Patent No. 0220414; 2002.

7. AFNOR. Essais des Eaux-Détermination de la Demande Chimique en Oxygène (DCO). Paris: AFNOR; 1988:Norme française NF T $90-101$.

8. Wagner W, Kruse A. Properties of Water and Steam. Berlin: Springer; 1998.

9. Villermaux J. Génie de la Réaction Chimique-Concept et Fonctionnement des Réacteurs. Paris: Tec\&Doc-Lavoisier; 1993.

10. Kodra D, Balakotaiah V. Modeling of oxidation of aqueous waste in a deep well reactor. AIChE J. 1992;38(7):988-1002.

11. Idel'cik IE. Memento des Pertes de Charge. Paris: Editions Eyrolles; 1986.

12. Chassaing P. Turbulence en mécanique des fluides: analyse du phénomeǹe en vue de sa modélisation à l'usage de l'ingénieur. Mécanique des Fluides-Eléments d'un Premier Cours. Collection Polytech, Toulouse: Ed. Cepaduès; 2000.

13. Patankar V. S. Numerical Heat Transfer and Fluid Flow. Levittown, NY: Taylor \& Francis; 1980.

14. Matéos D, Portela JR, Mercadier J, Marias F, Marraud C, Cansell F. New approach for kinetic parameters determination for hydrothermal oxidation reaction. Journal of Supercritical Fluids, in press.

15. Tester JW, Webley PA, Holgate HR. Revised global kinetic measurements of methanol oxidation in supercritical water. Industrial \& Engineering Chemistry Research. 1993;32(1):236-239.

16. Rofer CK, Streit GE. Oxidation of hydrocarbons and oxygenates in supercritical water, Phase II Final Report. Los Alamos National Laboratory, LA-11700-MS, DOE/HWP-90; 1989.

17. Webley PA, Tester JW. Fundamental kinetic of methanol oxidation in supercritical water. In: Johnston KP, Penninger JML, eds. Supercritical Fluid Science and Technology, ACS Symp. Ser. 406. Washington, DC: American Chemical Society; 1989:259-275. 